Mol. Cryst. Liq. Cryst., 1991, Vol. 202, pp. 17-25

Reprints available directly from the publisher

Photocopying permitted by license only

(C) 1991 Gordon and Breach Science Publishers S.A.

Printed in the United States of America

\title{
Adhesion of Vesicles and Membranes
}

\author{
REINHARD LIPOWSKY $\dagger$ and UDO SEIFERT $\ddagger$ \\ Sektion Physik der Universität München, Theresienstr. 37, 8000 München 2, West-Germany
}

(Received July 26, 1990)

\begin{abstract}
In the presence of an attractive surface, a vesicle can undergo shape transformations between two different free states, between a free and a bound state, and between two different bound states. Adhesion can also lead to topological changes such as vesicle rupture and vesicle fusion. The interaction between the vesicle membrane and the surface is renormalized by thermally excited shape fluctuations. This renormalization leads to unbinding phenomena both for fluid and for polymerized (or solid-like) membranes.
\end{abstract}

Keywords: shape transformation, vesicle, vesicle fusion, unbinding phenomena

\section{INTRODUCTION}

The mutual adhesion of membranes such as lipid bilayers can be experimentally studied by a variety of methods: (i) For oriented multilayer systems, the separation of the membranes can be measured by X-ray diffraction as a function of the external pressure $^{1}$; (ii) For membranes immobilized on mica surfaces, their direct interaction can be determined by the surface force apparatus ${ }^{2}$; (iii) Adhesion of giant unilamellar vesicles can be studied by micropipet aspiration techniques ${ }^{3}$; and (iv) Accidental adhesive contacts of membranes in diluted systems can be observed by light microscopy. ${ }^{4}$

The adhesion energy per unit area, $W$, deduced from these experiments is typically of the order of $\left(10^{-17}-10^{-15}\right) \mathrm{J} / \mu \mathrm{m}^{2}$ in case (i)-(iii) and of the order of $\left(10^{-20}-10^{-18}\right) \mathrm{J} / \mu \mathrm{m}^{2}$ in case (iv). Thus, the experimentally accessible range of adhesion energies extends over several decades.

In this paper, we briefly review recent theoretical work on the adhesion of vesicles and membranes. First, we consider the adhesion of vesicles to another surface, not necessarily another membrane. Within a simple model, the vesicle is predicted to undergo a variety of shape transformations between free and bound states, and between different bound states, ${ }^{5,6}$ see Sec. II.A. The adhesion process may also

† Address after 1 September 1990: Institut für Festkörperforschung, Forschungszentrum Jülich, Postfach 1913, 5170 Jülich, Germany.

\$ Address after 1 October 1990: Department of Physics, Simon Fraser University, Burnaby, B.C. V5A 1S6, Canda. 
induce topological changes of the vesicle such as vesicle rupture, see Sec. II.B, and vesicle fusion, see Sec. II.C.

The effective value, $W$, of the adhesion energy per unit area depends on the interplay of direct interactions and thermally excited shape fluctuations of the membranes. The latter fluctuations which are discussed in Sec. III.A act to reduce the value of $W$. The resulting unbinding phenomena can be studied by renormalization group methods, ${ }^{7,8}$ see Sec. III.B, and by Monte Carlo simulations, ${ }^{9,10}$ see Sec. III.C.

\section{ADHESION OF VESICLES}

\section{A. Adhesion by a Contact Potential}

Consider a vesicle which adheres to another membrane or surface. The shape of such a vesicle is determined by the interplay of adhesion and bending energies. This interplay can be theoretically studied starting from a relatively simple model in which the membrane experiences a contact potential arising from the attractive surface.

For a vesicle with total surface area, $A$, and enclosed volume, $V$, this model is defined by the free energy functional ${ }^{5}$

$$
F=\frac{1}{2} \kappa \oint d A\left(C_{1}+C_{2}\right)^{2}+\kappa_{g} \oint d A C_{1} C_{2}-W A^{*}+P V+\Sigma A
$$

The first two terms depending on the bending rigidity, $\kappa$, and on the Gaussian curvature modulus, $\kappa_{g}$, represent the curvature energies which are expressed in terms of the two principal curvatures, $C_{1}$ and $C_{2} \cdot{ }^{11}$ For simplicity, we focus on the case of zero spontaneous curvature.

The third term, $-W A^{*}$, in (2.1) represents the adhesion free energy of the membrane segment adjacent to the surface with contact area $A^{*}$. The last two terms in (2.1) involve the pressure difference $P=P_{e x}-P_{i n}$ and the lateral tension, $\Sigma$. First, we will focus on shape transformations which are fast compared to the permeation of water through the membrane. This situation is described by the ( $V$, $A$ )-ensemble for which $P$ and $\Sigma$ denote Lagrange multipliers.

For a given set of parameters, the most relevant vesicle state is the ground state, i.e., the state of lowest energy. It is convenient to introduce the dimensionless parameters $w \equiv W R^{2} / \kappa$ and $v \equiv V /\left((4 / 3) \pi R^{3}\right)$, with $R \equiv(A / 4 \pi)^{1 / 2}$. The corresponding phase diagram is shown in Figure 1. This phase diagram is divided into two parts by the lines $\left(D_{a}^{p r}\right),\left(D_{a}^{o b}\right),\left(D_{a}^{s t}\right)$, and $\left(C_{a}^{s t}\right)$ which represent the locus of transitions, $w=w_{a}(v)$, between free and bound states of the vesicle. For $w>$ $w_{a}(v)$, the state of lowest energy is bound while, for $w<w_{a}(v)$, it is free even in the presence of an attractive wall.

The bending energy is scale invariant, i.e., independent of the size of the vesicle, while the gain in adhesion energy is proportional to the area of the vesicle. Therefore, the critical value of the contact potential, $W=W_{a}=w_{a} \kappa / R^{2}$, is also size- 


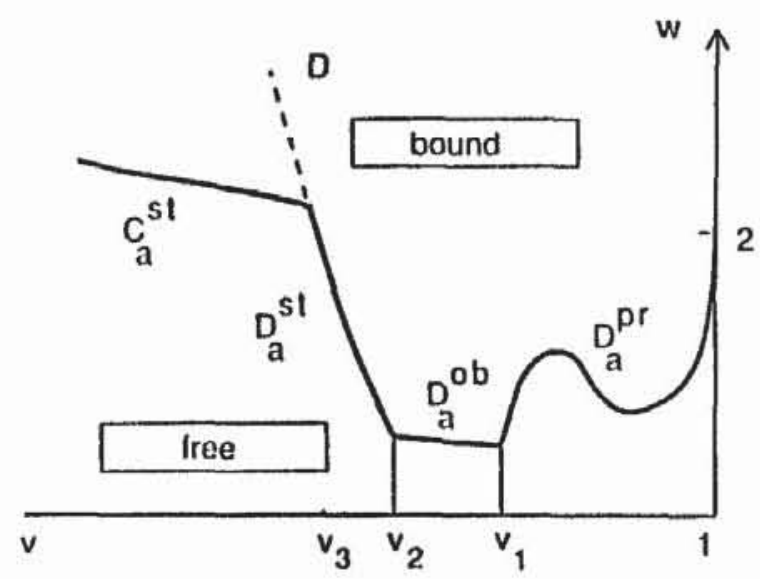

FIGURE 1 Schematic phase diagram for the shape transformations of a vesicle in the presence of an attractive surface. The thick line represents transitions between a free and a bound state, the thin full lines between two different free states, and the thin broken line $(D)$ between two different bound states. The parameters $w$ and $v$ are the reduced adhesion energy and the reduced volume as defined in the text.

dependent. Thus, for fixed $W$, vesicles of linear size $R>R_{a} \equiv\left(w_{a} \kappa / W\right)^{1 / 2}$ are bound while those of linear size $R<R_{a}$ are free.

For a single vesicle, the linear size $R=(A / 4 \pi)^{1 / 2}$ can be varied, to a certain extent, by a change in temperature since the thermal expansivity of the membrane surface is large compared to that of the enclosed water. This effect has been recently used in order to induce shape transformations of free vesicles: a variety of transformations has been experimentally observed and has been found to be in good agreement with theoretical calculations. ${ }^{12}$

For adhering vesicles, similar experiments would be highly valuable. One particularly promising method seems to be reflection interference contrast microscopy which has already been used for the shape of red blood cells. ${ }^{13}$ In this way, one might be able to observe how a single vesicle unbinds from the surface as the temperature is decreased since its linear size shrinks from $R>R_{a}$ to $R<R_{a}$. In Figure 2, two such shape sequences are shown as calculated theoretically.

Alternatively, let us consider, at fixed temperature, a dispersion of vesicles with different values of $V$ and $A$. If the vesicles stick to the surface for a long time, water can permeate through the membrane and the vesicle can eventually attain a state with zero pressure difference. Such 'ripe' vesicles are described by the $(P$, A)-ensemble with $P=0$. In this case, vesicles are bound for $w=W R^{2} / \kappa>2$ and free for $w \leqslant 2 .{ }^{5}$ Therefore, the size distribution of the bound vesicles will exhibit a lower cutoff at $R=R_{a}=(2 \mathrm{\kappa} / W)^{1 / 2}$.

\section{B. Vesicle Rupture Induced by Adhesion}

In the limit of strong adhesion, i.e., for large $w$ in Figure 1, the bound vesicle with constant volume (or constant pressure difference $P \leqslant 0$ ) attains the shape of a spherical cap. In this limit, $W$ is related to the lateral tension $\Sigma$ via the YoungDupre equation, $W=\Sigma\left(1+\cos \psi_{\text {eff }}\right)$, where $\psi_{\text {eff }}$ denotes an effective contact angle. ${ }^{5,6}$ If the tension $\Sigma$ exceeds a certain threshold, $\Sigma_{\max }$, it will disrupt the membrane. Thus, very strong adhesion with $W \geq \Sigma_{\max }$ will always lead to vesicle 


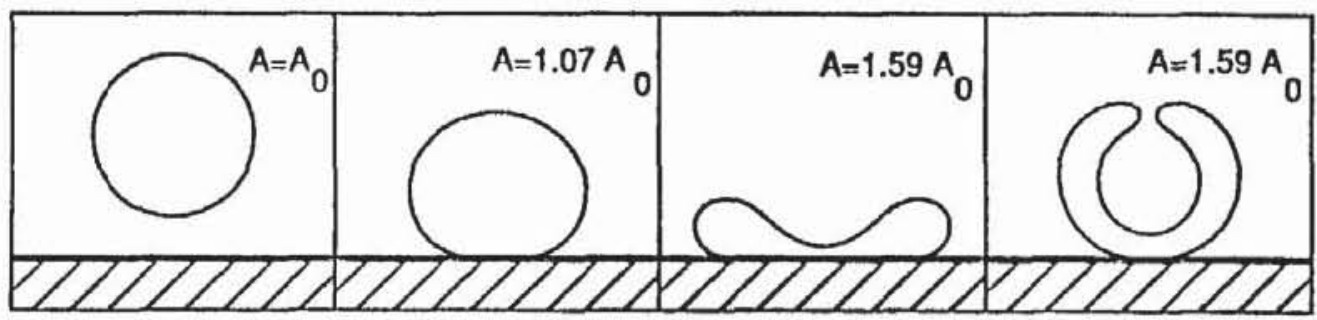

(a)

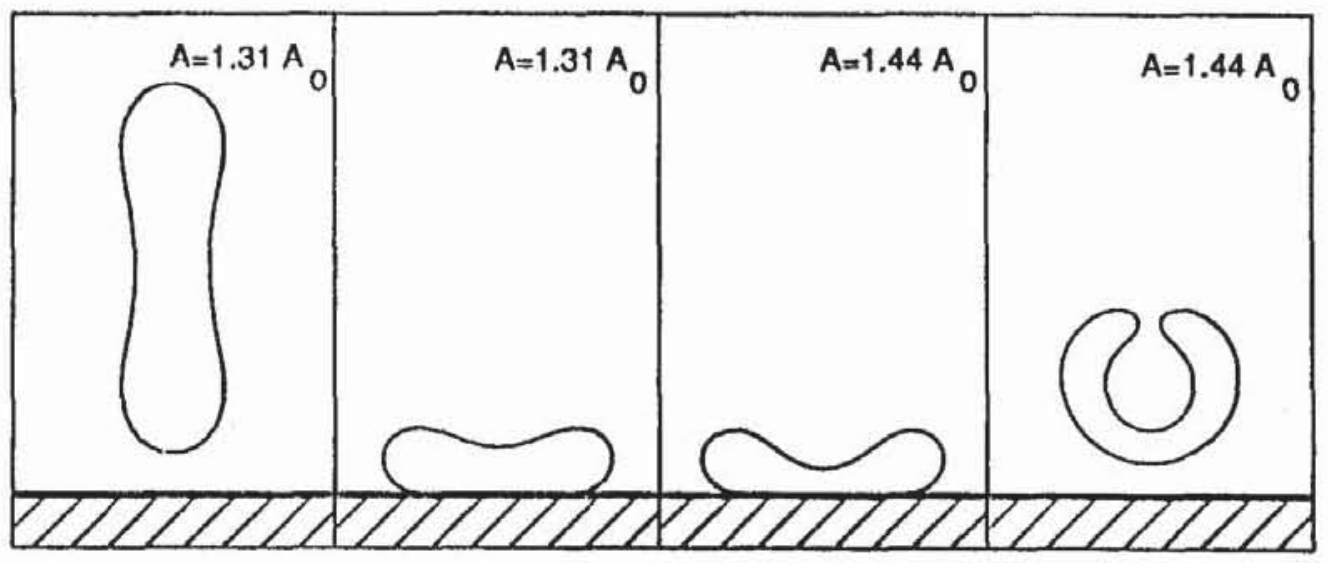

(b)

FIGURE 2 Shape transformations induced by a change in vesicle area, $A$, while the vesicle volume is kept constant. The initial state is a sphere with area $A_{o}$ and linear size $R_{o} \equiv\left(A_{0} / 4 \pi\right)^{1 / 2}$. (a) Surface with $W=1.8 \mathrm{\kappa} / R_{0}^{2}$ : The free vesicle becomes weakly prolate, then binds to the surface and finally undergoes a transition between two different bound states; and (b) Surface with $W=0.5 \kappa / R_{0}^{2}$ : the free vesicle becomes strongly prolate before it binds to the wall but then unbinds again as the area is further increased.

rupture. As a result, the closed vesicle will be transformed into a disk-like membrane segment with a free edge. This edge is characterized by an edge free energy per unit length or edge tension, $\Sigma_{e}$, arising from the partial contact between the hydrocarbon chains and the water.

The energy, $E_{f d}$, of a free planar disk with radius, $2 R$, is $E_{f d}=4 \pi R \Sigma_{e}$. On the other hand, if this disk is bound to the attractive surface, its energy is given by

$$
E_{b d}(R)=-4 \pi R^{2} W+4 \pi R \Sigma_{e}=-4 \pi R \Sigma_{e}\left(R-R_{b d}\right) / R_{b d} .
$$

with the length scale $R_{b d} \equiv \Sigma_{e} / W$.

Obviously, the energy of a bound disk is always smaller than the energy of a free disk. Thus, the ground state of the membrane segment in the presence of an attractive surface can be a bound disk, a free vesicle, or a bound vesicle. We will again consider 'ripe' vesicles described by the $(P, A)$-ensemble with $P=0$.

Free vesicles with $P=0$ attain a spherical shape with energy $E_{f_{v}}=4 \pi(2 \kappa+$ $\left.\kappa_{g}\right) \cdot{ }^{14}$ Bound vesicles with $P=0$, on the other hand, have energy $E_{b v}=E_{b v}(R)$ with $E_{b v}(R)<E_{f v}$ for $R>R_{a}=(2 \kappa / W)^{1 / 2}$. Close to the transformation point with $w=w_{a}=2$, this energy behaves as $E_{b v}-E_{f v} \sim-\kappa \Delta w^{2}$ for small $\Delta w \equiv w-$ 
$2 .{ }^{5}$ For large $w$, the bound vesicle resembles a flat pancake. In this limit, its energy behaves as

$$
E_{b v}(R)-4 \pi \kappa_{g}=-2 \pi R^{2} W+2 \pi g \sqrt{2 \kappa W} R+\mathcal{O}\left(w^{0}\right)
$$

with $g \simeq 2.8$.

In general, a comparison of the energies $E_{f v}, E_{b v}(R)$, and $E_{b d}(R)$ leads to several regimes which depend on the relative size of the two length scales $R_{a}=$ $(2 \kappa / W)^{1 / 2}$ and $R_{b d}=\Sigma_{e} / W$ (and on the value of $\kappa_{g}$ ). On the other hand, for sufficiently large vesicles with $R \gg R_{b d}=\Sigma_{e} / W$, one always enters a bound disk regime. As an example, consider phospholipid bilayers with $\mathrm{\kappa}=10^{-19} \mathrm{~J}$ and $\Sigma_{e} \simeq$ $5 \times 10^{-20} \mathrm{~J} / \mathrm{nm}$. For relatively strong adhesion with $W \simeq 10^{-16} \mathrm{~J} / \mu \mathrm{m}^{2}$, one has $R_{a}$ $\simeq 0.05 \mu \mathrm{m}$ and $R_{b d} \simeq 0.5 \mu \mathrm{m}$; for relatively weak adhesion with $W=10^{-20} \mathrm{~J} /$ $\mu \mathrm{m}^{2}$, these two scales are given by $R_{a} \simeq 5 \mu \mathrm{m}$ and $R_{b d} \simeq 0.5 \mathrm{~cm}$.

\section{Vesicle Fusion Induced by Adhesion}

So far, isolated vesicles or disks have been discussed. Now, let us imagine to increase the surface coverage, e.g., by increasing the bulk concentration of the lipid. It will then happen that bound vesicles (or disks) come into contact and fuse.

First, consider the possible fusion of two vesicles. For two free vesicles (with $P$ $=0$ ), the curvature model predicts that the energy change of fusion is given by ${ }^{14}$

$$
\Delta E_{f v}=2 E_{f v}-E_{f v}=4 \pi\left(2 \kappa+\kappa_{g}\right)
$$

Thus, fusion is energetically favorable for $\kappa_{g}>-2 \kappa$. Quite generally, adhesion acts to increase this energy change. Consider two identical vesicles of equal size, $R=(A / 4 \pi)^{1 / 2}$, which are bound to an attractive surface with $W>W_{a}=2 \kappa / R^{2}$. Then, the energy change of fusion is given by

$$
\Delta E_{b v}(R)=2 E_{b v}(R)-E_{b v}(\sqrt{2} R) .
$$

It then follows from (2.3) that

$$
\Delta E_{b v}(R) \approx 4 \pi g(\sqrt{2}-1) \sqrt{\kappa W} R+O\left(w^{0}\right)+4 \pi \kappa_{g}
$$

for large $R$ (or large $w$ ) with $g \simeq 2.8$ as in (2.3). Thus, in this limit, $\Delta E_{b v}$ is positive irrespective of the sign of $\kappa_{g}$. Furthermore, a numerical calculation shows that $\Delta E_{b v}(R)>\Delta E_{b v}\left(R_{a}\right)=\tilde{g} \pi \kappa+4 \pi \kappa_{g}>\Delta E_{f v}$ with $\tilde{g} \simeq 8.3$. Therefore, two vesicles can fuse in their bound state even if they cannot fuse in their free state because $\kappa_{g}<-2 \kappa$.

Fusion of two free vesicles is a thermally activated process which could start with the formation of two pores and a passage connecting them. The same mechanism is likely to apply to the fusion of two bond vesicles. There are, however, several factors which act to enhance the fusion rate: (i) For relatively large surface coverage, the probability for two vesicles to come into contact is strongly enhanced; (ii) Adhesion increases the lateral tension, $\Sigma$, within the membrane. In principle, this 
reduces the energy for pore formation since a pore of radius $L$ within a planar membrane has the energy $E_{\text {pore }}=2 \pi L \Sigma_{e}-\pi L^{2} \Sigma{ }^{15}$ For phospholipid bilayers, this reduction is, however, very small for the accessible values of $\Sigma$; and (iii) The surface may contain impurities or defects which can act as nucleation sites for the pore formation.

If the linear size of the bound vesicles grows by fusion, one will eventually enter the bound disk regime. If the edges of two disks come into contact, they will fuse immediately since no activation barrier is involved in such a process. Therefore, the average disk size for an ensemble of bound disks will steadily increase with time. For fixed surface coverage, such an ensemble of disks resembles an ensemble of droplets in the 2-dimensional Ising model with a conserved order parameter. In the latter situation, the average size of the disks should grow as $t^{1 / 3}$ with time $t$ for the late stages of the fusion process. ${ }^{16}$

\section{ADHESION AND UNBINDING TRANSITIONS}

The overall shape of a bound vesicle as discussed in the previous section can be observed through a light microscope. Now, let us imagine to use a microscope with a much larger resolution and let us focus on the region of contact between the vesicle and the second surface. Within this contact region, the two surfaces are separated by a small water gap and experience a variety of direct interactions arising from the intermolecular forces. Quite generally, these direct interactions are renormalized by thermally excited shape fluctuations of the membranes.

In fact, shape fluctuations with a wavelength of $1-10 \mu \mathrm{m}$ are even visible in the light microscope, see, e.g., Reference 13. It is important to realize, however, that there are many more length scales involved in the shape fluctuations. Indeed, the smallest wavelength of the membrane fluctuations is set by the size of the lipid molecules which is of the order of nm's. Thus, the shape fluctuations are composed of many modes with wavelengths from the molecular size up to the membrane dimension. These fluctuations lead to a certain membrane roughness. ${ }^{17}$

\section{A. Shape Fluctuations of Fluid and Polymerized Membranes}

For a rough state, the transverse displacements of the membrane from a reference plane can be regarded as an ensemble of humps: a membrane segment of linear size $L$ will form a hump of longitudinal and transverse extension, $L_{\|}$and $L_{\perp}$. These two length scales are related by

$$
L_{\|} \sim L \text { and } L_{\perp} \sim \mathscr{A} L_{\|}^{\zeta} \sim \mathscr{A} L^{\zeta} \quad \text { with } 0 \leq \zeta \leq 1
$$

where the amplitude $\mathscr{A}$ has the dimension of (length) ${ }^{1-5}$. This relation is invariant under the rescaling transformation given by

$$
L_{\|} \rightarrow L_{\|} / b \text { and } L_{\perp} \rightarrow L_{\perp} / b^{\zeta}
$$


with arbitrary rescaling factor $b$, which expresses the basic scale invariance of the shape fluctuations.

For fluid membranes with bending rigidity $\kappa$, one has $\zeta=1$ and $\mathscr{A}=\sqrt{T / \kappa} .{ }^{17}$ For polymerized (or solid-like) membranes with shear modulus $\mu$ and area compressibility modulus $K_{A}$, the roughness exponent satisfies $\zeta<1,7,18$ and the amplitude $\mathscr{A}$ in (3.1) is given by $\mathscr{A}^{2}=T^{\zeta} / \mathrm{K}^{2 \zeta-1} Y^{1-\zeta}$ with the Young modulus $Y \equiv 4$ $\mu K_{A} /\left(\mu+K_{A}\right)$ as follows from general scaling arguments. ${ }^{10}$ Computer simulations of tethered networks gave the effective exponent $\zeta=0.65 \pm 0.04 .{ }^{19-21}$ In contrast, our recent Monte Carlo simulations of elastic sheets showed that $\zeta=1 / 2{ }^{10}$

\section{B. Renormalization Group Approach to Unbinding Transitions}

As mentioned, the shape fluctuations renormalize the direct interaction and increase its repulsive part. The renormalized interaction may be attractive or repulsive at large membrane separation corresponding to a bound or an unbound state of the membranes. These two different states are separated by a phase boundary at which the membranes undergo an unbinding or adhesion transition. Such transitions were first predicted on the basis of renormalization group (RG) calculations ${ }^{7,8,22}$; their existence has been confirmed by Monte Carlo simulations ${ }^{9,10}$ and by experiments with sugarlipid membranes. ${ }^{23}$

The RG work implies that two lipid bilayers interacting with realistic intermembrane forces undergo such a transition at a critical unbinding temperature, $T=$ $T_{u}$. Alternatively, one may vary any parameter of the interaction such as, e.g., the Hamaker constant, $\bar{W}$, of the van der Waals interaction, see (3.4) below. In general, the RG leads to several universality classes or scaling regimes and thus to complex critical behavior. ${ }^{7,8}$ However, for sufficiently short-ranged interactions, one enters the strong fluctuation regime for which the unbinding transitions are predicted to be continuous and characterized by universal critical exponents. For example, the mean separation, $\langle\ell\rangle$, of the membrane from the other surface grows as

$$
\langle\ell\rangle \sim 1 /\left(T_{u}-T\right)^{\psi} \sim 1 /\left(\bar{W}-\bar{W}_{u}\right)^{\psi}
$$

as the unbinding temperature, $T_{u}$, or the critical value, $\vec{W}_{u}$, of the Hamaker constant is approached. The critical exponent $\psi$ is independent of the parameters of the direct interaction, and has the presumably exact value $\psi=1$ for fluid membranes, and the value $\psi \simeq 0.69<1$ for polymerized membranes. ${ }^{7}$

A lateral tension, $\Sigma>0$, acts to suppress continuous unbinding transitions. ${ }^{8}$ Indeed, such a tension always represents a relevant perturbation at the RG fixed points for such transitions. ${ }^{24}$ On the other hand, in the limit of small $\Sigma$, the surfaces attain a finite separation for $T<T_{u}$ but continuously unbind for $T \geq T_{u}$. Scaling arguments then show that the mean separation $\langle\ell\rangle \sim 1 / \Sigma^{1 / 2}$ while the adhesion free energy has a singular part $\sim-\Sigma^{1 / \zeta}$ as $\Sigma$ goes to zero for $T \geq T_{u}{ }^{6}$ For fluid membranes with $\zeta=1$, this behavior has been previously obtained by different arguments. $^{4}$

Another type of perturbation which could affect the unbinding behavior is the presence of frozen or quenched disorder. In this case, scaling arguments show that this perturbation is irrelevant provided the roughness exponent $\zeta$ and the unbinding 
exponent $\psi$ satisfy the relation $\zeta<\psi \cdot{ }^{6,8}$ For fluid membranes, one has $\zeta=\psi=$ 1 and thus a marginal case. Then, the critical behavior will presumably exhibit confluent logarithmic singularities in the presence of quenched disorder. For polymerized membranes with $\zeta=1 / 2$ and $\psi \approx 0.69$, such disorder should be irrelevant. $^{25}$

\section{Monte Carlo Simulations of Unbinding Phenomena}

The existence of unbinding transitions has been confirmed by Monte Carlo simulations. ${ }^{9,10}$ An example is shown in Figure 3. In this case, two fluid membranes with bending rigidities $\kappa_{1}=\kappa_{2}=0.2 \times 10^{-19} J$ have been studied. At separation $\ell$, these membranes experience the realistic interaction as given by

$$
\begin{aligned}
V(\ell)=P \ell+(\bar{W} / 12 \pi)\left[1 /\left(\ell^{2}+\ell_{0}^{2}\right)\right. & -2 /\left(\ell+\ell_{M}\right)^{2} \\
& \left.+1 /\left(\ell+2 \ell_{M}\right)^{2}\right]+B \exp \left(-\ell / \ell_{H}\right) .
\end{aligned}
$$

The parameter $P$ represents the external pressure, and $\bar{W}$ is the Hamaker constant of the van der Waals forces. For the empirical hydration interaction, the values $B$ $=0.2 \mathrm{~J} / \mathrm{m}^{2}$ and $\ell_{H}=0.3 \mathrm{~nm}$ have been chosen. Furthermore, we used the cutoff scale $\ell_{0}=\ell_{H}$, and the membrane thickness $\ell_{M}=4 \mathrm{~nm}$.

In Figure 3, the mean separation, $\langle\ell\rangle$, of the membranes is shown at room temperature as a function of external pressure, $P .{ }^{26}$ The different sets of Monte Carlo data correspond to different values of the Hamaker constant, $\bar{W}$. For comparison, the behavior at zero temperature, i.e., in the absence of shape fluctuations, is also included in Figure 3. Extrapolation of these and similar data to zero pressure

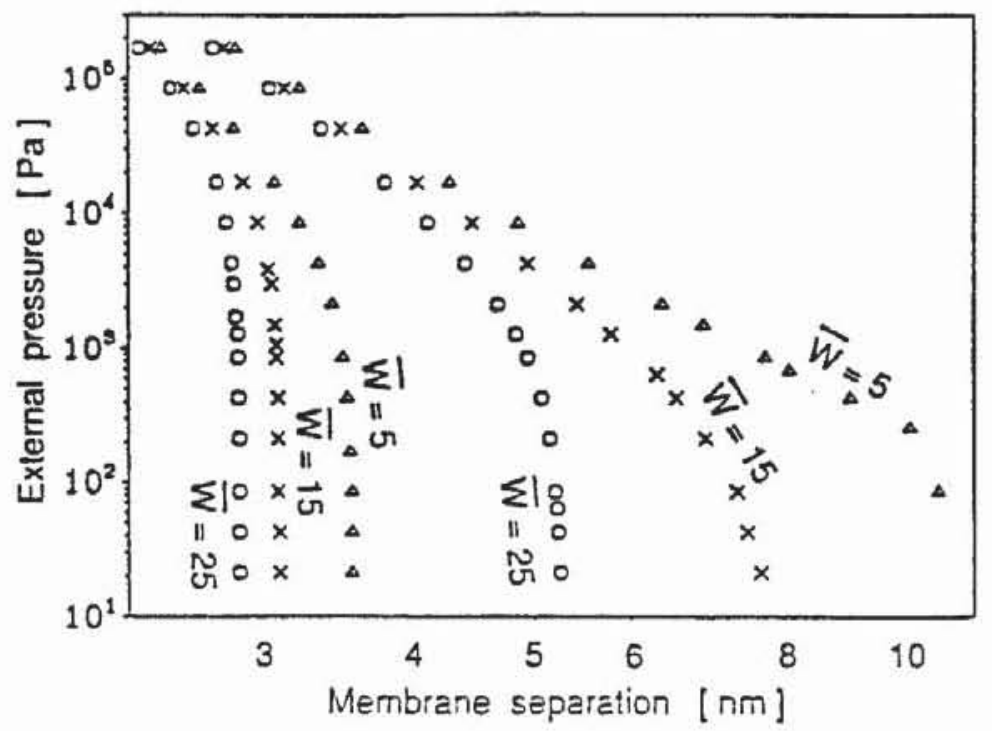

FIGURE 3 Monte Carlo data for the separation of two lipid bilayers plotted versus external pressure. The membranes have bending rigidities $\mathrm{\kappa}=0.2 \times 10^{-19} \mathrm{~J}$, and experience the interaction given by (3.4). The three curves on the right with Hamaker constants $\bar{W}=5,15$, and $25 \times 10^{-21} J$ are taken at room temperature; the three curves on the left represent the behavior in the absence of shape fluctuations. The difference between these two sets of curves shows the strong renormalization of the direct interaction by shape fluctuations. 
gives the critical value $\bar{W}=\bar{W}_{u} \simeq 3 \times 10^{-21} J$ for the Hamaker constant. Thus, attractive van der Waals interactions with $0<\bar{W} \leq \bar{W}_{u}$ are unable to bind the membranes as the pressure is decreased to zero.

\section{Acknowledgments}

We acknowledge support by the Sonderforschungsbereich 266 der Deutschen Forschungsgemeinschaft and by the Höchstleistungsrechenzentrum of the Forschungszentrum Jülich.

\section{References}

1. For a review, see R. P. Rand and V. A. Parsegian, Biochim. Biophys. Acta, 988, 351 (1989).

2. J. Marra and J. N. Israelachvili, Biochem, 24, 4608 (1985); J. Marra, J. Coll. Interf. Sci., 109, 11 (1986).

3. For a review, see E. Evans, Colloids and Surfaces, 43, 327 (1990).

4. R. M. Servuss and W. Helfrich, J. Phys. France, 50, 809 (1989); W. Harbich and W. Helfrich, J. Phys. France, 51, 1027 (1990).

5. U. Seifert and R. Lipowsky, Phys. Rev. A, 42, 4768 (1990).

6. R. Lipowsky and U. Seifert, in Fluctuations in lamellae and membranes, ed. by W. J. Benton and L. A. Turkevich, (Langmuir, to be published).

7. R. Lipowsky, Europhys. Lett., 7, 255 (1988), and Phys. Rev. Lett., 62, 704 (1989).

8. S. Grotehans and R. Lipowsky, Phys. Rev. A, 41, 4574 (1990).

9. R. Lipowsky and B. Zielinska, Phys. Rev. Lett., 62, 1572 (1989).

10. R. Lipowsky and M. Girardet, Phys. Rev. Lett., 65, 2893 (1990).

11. W. Helfrich, Z. Naturforsch. 28c, 693 (1973).

12. K. Berndl, J. Käs, R. Lipowsky, E. Sackmann and U. Seifert, Europhys. Lett., 13, 659 (1990).

13. A. Zilker, H. Engelhardt and E. Sackmann, J. Physique, 48, 2139 (1987).

14. W. Helfrich and W. Harbich, in Physics of Amphiphilic Layers, ed. by J. Meunier, D. Langevin und N. Boccara, Springer Proc. in Physics, Vol. 21 (Springer-Verlag, 1987).

15. C. Taupin, M. Dvolaitzky and C. Sauterey, Biochem. 14, 4771 (1975).

16. This follows from classical Lifshitz-Slyozov scaling, see, e.g., D. A. Huse, Phys. Rev. B, 34, 7845 (1986).

17. In principle, sufficiently strong shape fluctuations could lead to crumpled states of membranes. The corresponding unbinding phenomena have been discussed by R. Lipowsky and A. Baumgärtner, Phys. Rev. A, 40, 2078 (1989) and in R. Lipowsky, Physica Scripta T, 29, 259 (1989). In practice, self avoidance is a very strong constraint which acts to suppress crumpling.

18. D. R. Nelson and L. Peliti, J. Physique, 48, 1085 (1987).

19. S. Leibler and A. Maggs, Phys. Rev. Lett., 63, 406 (1989).

20. J.-S. Ho and A. Baumgärtner, Europhys. Lett., 12, 295 (1990).

21. F. F. Abraham and D. R. Nelson, Science, 249, 393 (1990).

22. R. Lipowsky und S. Leiber, Phys. Rev. Lett, 56, 2541 (1986), and 59, 1983 (E) (1987).

23. M. Mutz and W. Helfrich, Phys. Rev. Lett., 62, 2881 (1989).

24. This also follows from results for wetting transitions, see R. Lipowsky, Phys. Rev. Lett., 52, 1429 (1984).

25. For direct interactions which exhibit several minima, the surfaces can undergo multicritical unbinding transitions at which quenched disorder should change the critical behavior, see Reference 8 .

26. R. Lipowsky and B. Zielinska, (unpublished). 